\title{
Recent Advances in Machine-to-Machine Communications
}

\author{
Nian Xia, Chu-Sing Yang \\ Institute of Computer and Communication Engineering, Department of Electrical and Engineering, National \\ Cheng Kung University, Taiwan \\ Email: brastme@gmail.com, csyang@ee.ncku.edu.tw
}

Received 24 January 2016; accepted 22 May 2016; published 29 May 2016

\begin{abstract}
In the near future, there are expected to have at least billions of devices interconnected with each other. How to connect so many devices becomes a big issue. Machine-to-Machine (M2M) communications serve as the fundamental underlying technologies to support such Internet of Things (IoT) applications. The characteristics and services requirements of machine type communication devices (MTCDs) are totally different from the existing ones. Existing network technologies, ranging from personal area networks to wide area networks, are not well suited for M2M communications. Therefore, we first investigate the characteristics and service requirements for MTCDs. Recent advances in both cellular and capillary M2M communications are also discussed. Finally, we list some open issues and future research directions.
\end{abstract}

\section{Keywords}

Machine-to-Machine Communications, IoT, MTC, Capillary M2M Communications, Cellular M2M Communications

\section{Introduction}

Machine-to-Machine (M2M) communications, also known as Machine-Type Communications (MTC), are a new communication paradigm, in which MTCDs can communicate with each other automatically without or with little human intervention. Internet of Things (IoT) refers to the interconnection and communication among different devices. M2M communications serve as the essential underlying communication technologies for IoT. M2M communications have been applied into many fields including: smart metering, security systems, m-health, intelligent transport systems, and so on.

According to the operational spectrum, M2M communications can be classified into two categories: cellular M2M communications and capillary M2M communications. Cellular M2M communications contain standards using the licensed spectrum resource such as LTE-A while capillary M2M communications use the unlicensed ISM bands such as Wi-Fi. Different from the traditional human-centric communications where most of the traffic is in the downlink, M2M communications have some unique traffic features: 1) small in size but high in frequency data transmissions [1]; 2) mostly uplink and delay-tolerant traffic; and 3) event-driven and periodic traffic. However, existing network protocols, ranging from wireless personal area networks (WPANs) to wide area networks (WANs), are all optimized for human-centric communications. Hence, they cannot fulfill the require- 
ments proposed by M2M communications. For example: the existing network protocols which are optimized for handling large and burst traffic is not efficient in handling the small but frequent data transmission. Although energy efficiency has gain more and more attentions in existing protocols, it is still not as important as the data rate and delay requirements. On the contrary, in M2M communications energy efficiency plays a more important rule than the data rate and delay requirements. The energy consumption in existing protocols is still too high for MTCDs. MTCDs are mostly battery-powered and required to live at most several years without replacing batteries. The number of MTCDs are expected to be at least tens of thousands within the cell range. No existing standards can handle so many MTCDs simultaneously.

These issues incurred by M2M communications all motivate the design of new protocols and standards. In this paper, we investigate several new protocols or enhancements of existing protocols for meeting the requirements proposed by MTCDs in both capillary and cellular M2M communications. In capillary M2M communications, we discuss two representative network protocols in personal area networks and local area networks respectively: Bluetooth Smart and Wi-Fi. In cellular M2M communications, LTE-A enhancements for MTC is considered.

The organization of this paper is as follows. In the next section, we discuss the modifications in the newly introduced Wi-Fi standard 802.11ah and Bluetooth Smart for supporting M2M communications. The enhancements in LTE-A for MTC are discussed in section 3. In section 4, we discuss several open issues and list several future research directions. We conclude our paper in section 5.

\section{Capillary M2M Communications}

Capillary M2M networks consist of several unlicensed band wireless technologies: Wi-Fi, Bluetooth, Zigbee, Sun, 802.15.6 and so on. The 802.15.x standards have been discussed for many years in sensor networks. In this section, we focus on Wi-Fi and Bluetooth which provide new standards specified for M2M communications.

\subsection{11ah}

The IEEE 802.11ah Task Group, creates in 2010, provided the 802.11ah standard for addressing the need for M2M communications in wireless local area networks. The 802.11ah belongs to the Wi-Fi standard family. The service requirements defined by 802.11ah to support M2M communications are shown below [2]:

- Up to 8191 MTCDs within an access point (AP).

- Carrier frequency of below $1 \mathrm{GHz}$ is less congested and guarantee a long range.

- Data rate of at least $100 \mathrm{kbps}$.

- One-hop network topologies.

- Short and small data transmission.

- Low energy consumption by power saving mechanisms.

MTCDs within an AP are divided into three different categories: traffic indication map (TIM) MTCDs, nonTIM MTCDs and unscheduled MTCDs [3]. MTCDs with large volume of data are recommended to deploy TIM MTCDs while unscheduled MTCDs are suitable for MTCDs with sporadic traffic. A new association identifier (AID) which can classify MTCDs into pages, TIM groups, sub-blocks and indexes in the sub-blocks is proposed and the 13bit AID can support at most 8191 MTCDs with unique AIDs. A four-layer hierarchical structure in AID not only save energy consumption of MTCDs in listening the channel, but also provide diverse services for different groups.

To prolong the life cycle of MTCDs, 802.11ah introduces several power saving mechanisms. First, the power saving mode where the radio module is turned off is activated during non-traffic periods. Second, the TIM and Segmentation scheme reduces the competing time of MTCDs for channels and increases the sleep time. Third, 802.11ah supports long sleep period (up to years) after the initial handshaking with AP. However, this will lead to synchronization problems. To support small data transmission, 802.11ah introduces three enhancements. First, 802.11ah shortens its header from 28 bytes to 18 bytes by using AID instead of MAC addresses. Second, 802.11ah defines several null data packet frames to shorten the current 802.11 signaling frames. Finally, a speed frame exchange scheme is introduced to replace the traditional ACK.

\subsection{Bluetooth Smart}

Bluetooth Special Interest Group (SIG) introduced Bluetooth Low Energy (BLE), also known as Bluetooth 
Smart, in Bluetooth 4.0 [4] for power-constrained MTCDs with small but frequent data transmissions. This is different from traditional Bluetooth applications, such as audio streaming which has relative large amounts of data transmission and frequent interactions between host and slave devices. The channel bandwidth increases from $1 \mathrm{MHz}$ to $2 \mathrm{MHz}$ in BLE to support higher data rates. Correspondingly, the total number of channels are reduced from 80 to 40 with three reserved for advertising channels and 37 for data channels.

Originally, Bluetooth only supports the start topology where a slave node can only connect to at most one master node. However, a slave node can connect to multiple master nodes after Bluetooth 4.1 [5] and mesh networks can be supported. IP stack is put into BLE to enable Bluetooth devices to connect the Internet and this makes BLE more suitable for IoT applications. BLE uses the following methods to reduce power consumption [6]:

- Keeping the packet short. The short packet can simplify the circuit design and reduce the peak power consumption. It should also be noted that the inter-packet gap time to cool down silicon further reduces power consumption.

- Using a high physical bit rate. A high data rate can reduce the time to transmit data which can reduce the energy consumption. Although a complex modulation scheme can increase the data rate, more power are needed to modulate and demodulate data. Hence, a simple modulation scheme with low peak-power consumption is more energy efficient.

- Providing low overhead. BLE does not require the acknowledgement of a packet immediately. It means that a BLE device can wait until it has packets to transmit before acknowledging the last packet transmitted.

- Optimized acknowledgement scheme.

- Single-channel connection events. A connection event is defined as all communications between a master node and a slave node. BLE stays on one frequency in a single-channel event, and hops to a new frequency when a next event occurs. This scheme can minimum the latency to transmit data and reduce energy consumption.

- Subrating connection events. BLE allows the slave devices to decrease the frequency of listening to the master devices to reduce energy consumption.

- Using offline encryption. The data encryption process consumes more power in real time cases. In BLE, encryption can take place before a packet is transmitted (offline) when the radio is off.

It is worth mentioning that BLE cannot fulfillall the requirements proposed by M2M communications. In other words, BLE only need to satisfy M2M requirements in personal area networks. BLE can support 127 devices and this is enough for any application in personal area networks. With the prosperity of wearable devices, BLE is regarded as a promising technology due to its low energy consumption and long lifetime. The number of connected MTCDs at the same time is not the major concern of BLE due to the limited serving range.

\section{Cellular M2M Communications}

In this section, we study LTE-A cellular network enhancements for supporting M2M communications. 3GPP first proposed services requirements for MTC in LTE-A in 2009 [7].

\subsection{LTE-A Systems}

LTE-A cellular systems designed for traditional Human-to-Human $(\mathrm{H} 2 \mathrm{H})$ communications primarily focus on high data rates and low delay requirements and these are not of equal important in M2M communications. In contrast, M2M communications focus on M2M specific requirements such as massive access, small data transmission and power saving.

The existing cellular network can support at most tens of UEs within a cell and this is not enough for M2M communications which may have thousands of MTCDs. To support massive access in LTE-A, several methods are proposed as follows:

- Access class barring [8]. 3GPP suggests to use separate access classes for MTC. Each MTCD is assigned to a class with independent rejection probabilities and backoff timers. Without the need for new classes, MTCDs configured for Extend access barring (EAB) will be restricted to access the network with a barring factor if congestion occurs.

- Slotted access. In the slotted access scheme, each MTCD can only transmit their random access requests during their assigned time slots. 
- Group-based access. MTCDs are divided into different groups and MTCDs within the same group have the same identity in the random access (RA) process. The number of requested preambles reduces to the number of groups.

- Increasing preambles. Adding preambles can also increase the number of serving MTCDs. The total number of preambles defined by 3GPP is 64 [9]. Apart of the reserved preambles for special purposes, the actual number of preambles that can be used in LTE-A is 54. A spatial group-based random access method to increase the number of preambles is proposed by Jang et al. in [10].

- Modifying RA procedure. The regular random access process contains four steps. The collision can only be detected in the third step. The traditional random access will not transmit message 4 in the fourth step once it detects collision in the third step. To decrease the collision probability, a modified message 4 containing the set of preambles to be used in the next contention attempt is transmitted.

To achieve power saving, LTE/LTE-A introduces the discontinuous reception (DRX) mechanism in the downlink for UEs. DRX has two different DRX cycles: short DRX cycles and long DRX cycles. However, the maximum short and long DRX cycle is $640 \mathrm{~ms}$ and 2.56s which is still too short for MTCDs with infrequent data transmissions. Authors in [11] proved that a longer DRX cycle can lead to significant gains in the energy consumption of MTCDs. A new power save mode (PSM) for MTC is introduced in LTE-A Rel-13. The location of some MTCDs are fixed and there is no need to do mobility management for these devices as usual UEs. Repetition code is used to extend the coverage for M2M communications in LTE-A.

\subsection{LTE-M}

Even with M2M features being added in LTE Rel-12, it is not optimized for M2M services [12]. A new device category Cat 0 was introduced in Rel-12 for MTCDs. Before Cat 0, LTE Cat 1 is recommended for M2M applications due to its narrow bandwidth and low data rate. The downlink and uplink peak rates for Cat 1 devices are $10 \mathrm{Mbps}$ and $5 \mathrm{Mbps}$ respectively while both the downlink and uplink peak rates for Cat 0 devices are 1 Mbps. A narrowband system has many advantages. First, narrow bandwidth requires less expensive RF components. Coverage can be extended because transmit power can concentrate in the narrow bandwidth. Finally, it can also improve the spectrum efficiency. Cat 0 devices use half duplex mode between uplink and downlink, and this approach can save energy consumption at the cost of delay.

\section{Open Issues and Research Challenges}

In this section, we discuss several open issues for M2M communications. Some research directions are also proposed.

\subsection{Coexistence Problems}

The capillary M2M communication protocols use the unlicensed ISM band which is crowd by many different protocols due to its open access nature. The interference from other protocols operating in the ISM band cannot be ignored when deploying capillary M2M communications. This is one reason why 802.11ah uses the less congested below $1 \mathrm{GHz}$ ISM bands rather than the common used $2.4 \mathrm{GHz}$ ISM bands. However, Bluetooth, Zigbee, 802.15.6 and 802.11b use the same 2.4 GHz ISM. Moreover. So the interference problem is inevitable for these protocols. 3GPP recently proposed the LTE-U (operate LTE in unlicensed bands) and undoubtedly this will worsen the congestion in the ISM bands. How to mitigate the interference among different protocols becomes a critical problem. One optional way to mitigate interference is to use cognitive radio technologies to sense the unlicensed spectrum before using it.

The coexistence of MTCDs and Human-to-Human $(\mathrm{H} 2 \mathrm{H})$ devices is also an important problem. MTCDs should reuse as many radio resources as possible while not interrupting the traditional H2H devices. However, MTCDs will easily use up all the radio resources and block the $\mathrm{H} 2 \mathrm{H}$ traffic without a rough resource allocation method. A dynamic resource allocation method is needed to solve this problem.

\subsection{Scheduling in Heterogeneous Networks}

Heterogeneous networks consists of small cells, picocells and macro cells. A MTCD can either connect to an eNB directly, or through a small cell base stations. If possible, a MTCD can also use unlicensed band technolo- 
gies such as Bluetooth to access the network. Cellular technologies can provide delay, coverage and data rate guarantees at the cost of high power consumption. Capillary technologies such as Bluetooth can reduce energy consumption at the cost of high delay and low data rates. MTCDs equipped with multiple technologies need to determine which technology to use. A naive approach is to use the round-robin method between the available technologies. However, this approach is not the most energy efficient one. Choose a suitable technology to transmit data based on the applications is a candidate method. For applications with strict delay requirements, we prefer to use delay-guaranteed technologies such as cellular M2M. For delay-tolerant applications, we can use technologies which can decrease the energy consumptions such as BLE.

\subsection{Ultra-Dense Networks}

In the future, MTCDs deployed densely in a small region will form an ultra-dense network. All the MTCDs cannot connect to one central node (eNB or AP) as it does in normal networks and efficient offloading methods are needed to do load balance. The propagation model is totally different with existing ones in ultra-dense networks, and new propagation models are also needed. Mobility management for ultra-dense networks is also an important problem that needs to be addressed. How to do mobility management in ultra-dense networks is still a research challenge.

\section{Conclusion}

The convergence of different types of technologies in M2M communications pose great challenges to both academic and industry. In this paper, we want to bridge the gap between M2M capillary and cellular communications to give readers a broad view of M2M communications. We first investigate new characteristics and service requirements incurred by MTCDs. Recent advances in both capillary and cellular M2M communications for fulfilling the requirements are discussed. Finally, we discuss several open issues and research challenges for researchers interested in this field.

\section{Acknowledgements}

This work was supported in part by Taiwan Ministry of Science and Technology (MOST) under Grants MOST 104-2218-E-001-002- and MOST 103-2221-E-006-145-MY3.

\section{References}

[1] 3GPP TR 23.887 (2014) Study on Machine-Type Communications (MTC) and Other Mobile Data Applications Communications Enhancements (Release 12).

[2] Aust, S., Prasad, R.V. and Niemegeers, I.G.M.M. (2012) IEEE 802.11ah: Advantages in Standards and Further Challenges for Sub $1 \mathrm{GHz}$ Wi-Fi. IEEE International Conference on in Communications (ICC), 6885-6889.

[3] Adame, T., Bel, A., Bellalta, B., Barcelo, J. and Oliver, M. (2014) IEEE 802.11AH: The WiFi Approach for M2M Communications. IEEE Wireless Communications, 21, 144-152. http://dx.doi.org/10.1109/MWC.2014.7000982

[4] Bluetooth Special Interest Group (2010) Bluetooth Core Specification Version 4.0.

[5] Bluetooth Special Interest Group (2013) Bluetooth Core Specification Version 4.1.

[6] Heydon, R. (2012) Bluetooth Low Energy. The Developers Handbook. Prentice Hall, Englewood Cliffs.

[7] 3GPP TS 22.368 (2009) Service Requirements for Machine-Type Communications (Release 10).

[8] 3GPP TR 37.868 (2011) Study on RAN Improvements for Machine Type Communications (Releases 11).

[9] 3GPP TS 36.211 (2015) Evolved Universal Terrestrial Radio Access Network: Physical Channels and Modulation (Release 12).

[10] Jang, H.S., Kim, S.M., Ko, K.S., Cha, J. and Sung, D.K. (2014) Spatial Group Based Random Access for M2M Communications. IEEE Communications Letters, 18, 961-964. http://dx.doi.org/10.1109/LCOMM.2014.2320970

[11] Tirronen, T., Larmo, A., Sachs, J., Lindoff, B. and Wiberg, N. (2012) Reducing Energy Consumption of LTE Devices for Machine-to-Machine Communication. IEEE Globecom, 1650-1656. http://dx.doi.org/10.1109/glocomw.2012.6477833

[12] Ratasuk, R., Mangalvedhe, N., Ghosh, A. and Vejlgaard, B. (2014) Narrowband LTE-M System for M2M Communication. IEEE Vehicular Technology Conference, 1-5. 